\title{
Equipos de protección individual para cuerpo frente a agentes biológicos en trabajadores sanitarios
}

\section{Personal protective equipment for the body against biological agents in healthcare workers}

\author{
Joan Inglés-Torruella ${ }^{1}$ \\ 1 Unitat de Vigilància de la Salut. Hospital Universitari Sant Joan. Reus (Tarragona), España.
}

\section{Resumen}

Este trabajo es un comentario del artículo: Verbeek JH, Rajamaki B, ljaz S, Sauni R, Toomey E, Blackwood B, Tikka C, Ruotsalainen JH, Kilinc Balci FS. Personal protective equipment for preventing highly infectious diseases due to exposure to contaminated body fluids in healthcare staff. Cochrane Database of Systematic Reviews. 2020;(5): Art. No.: CD011621. doi:10.1002/14651858.CD011621.pub5.

\begin{abstract}
This text is a commentary on the article: Verbeek JH, Rajamaki B, ljaz S, Sauni R, Toomey E, Blackwood B, Tikka C, Ruotsalainen JH, Kilinc Balci FS. Personal protective equipment for preventing highly infectious diseases due to exposure to contaminated body fluids in healthcare staff. Cochrane Database of Systematic Reviews. 2020;(5): Art. No.: CD011621. doi:10.1002/14651858.CD011621.pub5.
\end{abstract}

Sección coordinada por

Consol Serra (consol.serra@upf.edu) | Mª del Mar Seguí (mm.segui@ua.es)

Fechas · Dates

Recibido: 2020.07.02

Aceptado: 2020.07.12

Publicado: 2020.07.15

\section{Correspondencia $\cdot$ Corresponding Author}

Joan Inglés Torruella

jinglestorruella@gmail.com 


\section{Resumen del artículo comentado}

En las epidemias de enfermedades muy infecciosas como la enfermedad por el virus del Ébola, el Síndrome Respiratorio Agudo Severo (SARS) o el coronavirus (COVID-19), el personal sanitario (PS) corre un riesgo mucho mayor de infección que la población general debido al contacto con los fluidos corporales contaminados de los pacientes. Los equipos de protección individual (EPI) pueden reducir el riesgo al cubrir las partes expuestas del cuerpo. No está claro qué tipo de EPI protege mejor, cuál es la mejor manera de ponérselos y quitárselos, ni cómo capacitar a los trabajadores sanitarios en el uso adecuado del equipamiento.

Al objeto de evaluar qué tipo de EPI para todo el cuerpo y qué método para colocarlo o retirarlo tiene el menor riesgo de contaminación o infección para los trabajadores sanitarios, así como qué métodos de capacitación pueden mejorar el cumplimiento de los protocolos de utilización del EPI, se realizaron búsquedas bibliográficas en CENTRAL, MEDLINE, EMBASE y CINAHL hasta el 20 de marzo de 2020. Se incluyeron todos los estudios controlados que evaluaron los efectos de los EPI para protección del cuerpo, utilizados por los sanitarios expuestos a enfermedades altamente infecciosas, respecto al riesgo de infección o contaminación, o el incumplimiento de los protocolos. También se incluyeron los estudios que compararon el efecto de diversas maneras de colocarse o retirarse el EPI, así como los efectos de la capacitación de los sanitarios en estos mismos resultados. Dos autores de la revisión, de forma independiente, seleccionaron los estudios, extrajeron los datos y evaluaron el riesgo de sesgo en los ensayos incluidos. Cuando fue posible se realizaron metanálisis de efectos aleatorios.

Las versiones anteriores de esta revisión se publicaron en 2016 y 2019. En esta actualización se incluyeron 24 estudios con 2278 participantes, de los cuales 14 fueron ensayos controlados aleatorizados (ECA), uno fue un ensayo cuasi-aleatorizado y nueve tuvieron un diseño no aleatorizado. Ocho estudios compararon distintos tipos de EPI. Seis estudios evaluaron EPI adaptados. Ocho estudios compararon procedimientos para colocarse y retirarse el equipo y tres estudios evaluaron tipos de capacitación. Dieciocho estudios utilizaron la exposición simulada con marcadores fluorescentes o virus inocuos.

En los estudios de simulación, la mediana de las tasas de contaminación fue del $25 \%$ en el caso de la intervención y del $67 \%$ en los grupos control. La evidencia es de calidad muy baja para todos los resultados, a menos que se indique lo contrario, debido a que se basa en uno o dos estudios, a la evidencia indirecta en los estudios de simulación y al riesgo de sesgo.

En cuanto a los distintos tipos de EPI, el uso de un respirador eléctrico purificador del aire propulsado con un mono puede proteger contra el riesgo de contaminación mejor que una mascarilla N95 y una bata (riesgo relativo [RR] 0,27; intervalo de confianza [IC] del 95\%: 0,17 a 0,43), pero es más difícil de poner (incumplimiento: RR 7,5; IC del 95\%: 1,81 a 31,1). En un ECA (59 participantes) los monos de protección fueron más difíciles de quitar que las batas de aislamiento (evidencia de certeza muy baja). Las batas pueden proteger mejor contra la contaminación 
que los delantales (manchas pequeñas: diferencia de medias [DM] -10,28; IC del 95\%: -14,77 a -5,79). El EPI hecho de un material más transpirable puede provocar más puntos de contaminación en el tronco (DM 1,60; IC del 95\%: 0,15 a 3,35) en comparación con un material más hidrófugo, pero se puede asociar con una mayor satisfacción del usuario (DM -0,46; IC del 95\%: -0,84 a -0,08; escala del 1 al 5). Las modificaciones en el diseño de los EPI pueden dar lugar a una menor contaminación en comparación con los EPI estándar: combinación de bata y guante sellados (RR 0,27; IC del 95\%: 0,09 a 0,78), una bata con mejor ajuste alrededor del cuello, las muñecas y las manos (RR 0,08; IC del 95\%: 0.01 a 0,55), una mejor cobertura de la interfaz bata-muñeca (RR 0,45; IC del 95\%: 0,26 a 0,78, evidencia de certeza baja), incorporación de lengüetas para agarrar y facilitar la retirada de las mascarillas (RR 0,33; IC del 95\%: 0,14 a 0,80) o los guantes (RR 0,22; IC del 95\%: $0,15$ a 0,31$)$.

Seguir las recomendaciones de los Centers for Disease Control and Prevention (CDC) para retirarse el equipo puede dar lugar a menos contaminación en comparación con ninguna guía (manchas pequeñas: DM -5,44; IC del 95\%: -7,43 a -3,45). Quitarse los guantes y la bata en un solo paso puede dar lugar a menos contaminación bacteriana (RR 0,20; IC del 95\%: 0,05 a 0,77) pero no a menos contaminación fluorescente (RR 0,98; IC del 95\%: 0,75 a 1,28) que quitárselos por separado. El doble guante puede dar lugar a una menor contaminación viral o bacteriana en comparación con un guante solo (RR 0,34; IC del 95\%: 0,17 a 0,66), pero no a menos contaminación fluorescente (RR 0,98; IC del 95\%: 0,75 a 1,28). Las instrucciones habladas adicionales pueden dar lugar a menos errores durante la retirada del equipo (DM -0,9; IC del 95\%: - 1,4 a -0,4) y a menos puntos de contaminación (DM -5; IC del 95\%: -8,08 a -1,92). La limpieza adicional de los guantes con amonio cuaternario o lejía antes de quitarse el equipo puede disminuir la contaminación, pero no, frotarse las manos con alcohol.

El uso adicional de una simulación por ordenador para la capacitación del PS puede dar lugar a menos errores al quitarse el equipo (DM -1,2; IC del 95\%: -1,6 a $-0,7)$. Una videoconferencia sobre la colocación del EPI puede dar lugar a mejores puntuaciones en las capacidades (DM 30,70; IC del 95\%: 20,14 a 41,26) que una conferencia tradicional. La instrucción presencial puede reducir más el incumplimiento de la guía para quitarse el equipo (odds ratio 0,45; IC del 95\%: 0,21 a 0,98) que la provisión de folletos o vídeos solamente.

Se encontró evidencia de certeza baja a muy baja, que cubrir más partes del cuerpo puede conducir a una mejor protección, pero a menudo se hace a costa de que sea más difícil ponérselo o quitárselo y menos cómodo de llevar. Los tipos de EPI más transpirables pueden conducir a una contaminación similar, pero se pueden asociar con una mayor satisfacción del usuario. Las modificaciones en el diseño del EPI, como las lengüetas para agarrar, pueden disminuir el riesgo de contaminación. En el caso de los procedimientos de colocación y retirada de los guantes, seguir las guías proporcionadas por los CDC, retirarse los guantes y las batas en un solo paso, el uso de guantes dobles, las instrucciones habladas durante la retirada y la desinfección de los guantes pueden reducir la contaminación y aumentar 
el cumplimiento. La capacitación presencial en el uso del EPI puede reducir más los errores que la capacitación con folletos.

Aún se necesitan ECA de capacitación con seguimiento a largo plazo. Se necesitan estudios de simulación con más participantes para determinar qué combinaciones de EPI y qué procedimiento para quitárselo protegen mejor. Es urgente alcanzar un consenso sobre la simulación de la exposición y la evaluación de los resultados. También se necesita más evidencia de la práctica real. Por lo tanto, se debe registrar el uso de EPI por parte de los sanitarios expuestos a enfermedades muy infecciosas y se deben seguir de manera prospectiva con respecto al riesgo de infección.

\section{Comentario}

Se entiende por equipo de protección individual (EPI) aquellos equipos destinados a ser llevados o sujetados por un trabajador para protegerlo de uno o más riesgos que pueden amenazar su seguridad o salud en el trabajo, así como cualquier complemento o accesorio destinado a tal fin ${ }^{(1,2)}$. A éste efecto, la normativa aplicable es el Real Decreto 773/1997, de 30 de mayo, sobre disposiciones mínimas de seguridad y salud relativas a la utilización por los trabajadores de equipos de protección individual, y el Real Decreto 1407/1992, de 20 de noviembre, por el que se regulan las condiciones para la comercialización y libre circulación intracomunitaria de los equipos de protección individual(3). El INSHT dispone de una guía técnica para la interpretación del Real Decreto 773/1997(1) y la CEE también dispone de otra guía técnica para la interpretación de la Directiva 89/686 EEC ${ }^{(4,5)}$. Cuando el personal sanitario, en aplicación de la LPRL, está expuesto a un riesgo tal que hace necesario el uso de un EPI, de acuerdo con lo establecido en el Real Decreto 773/1997, el equipo debe estar certificado de acuerdo con el Real Decreto 1407/1992, lo que queda evidenciado por el marcado CE de conformidad(3).

Para combatir los riesgos de accidentes y de otros daños a la salud, resulta prioritaria la aplicación de medidas técnicas y organizativas destinadas a eliminar los riesgos en su origen o a proteger a los trabajadores mediante disposiciones de protección colectiva. Cuando estas medidas se revelan insuficientes, se impone la utilización de EPIs, a fin de prevenir los riesgos residuales ineludibles. El hecho de que se recurra a los EPIs como "último escalón" de la acción preventiva no se debe a que este tipo de medida sea menos importante que las demás. El principal motivo, para anteponer otro tipo de medidas a la protección individual, se debe a que la utilización de una protección individual supone establecer un contacto directo del trabajador con el riesgo, sin que exista otra "barrera" detrás para eliminar o disminuir las consecuencias del daño en caso de que el peligro se materialice $y$, en estas condiciones, si el EPI falla o resulta ineficaz, el trabajador sufrirá las consecuencias del daño con toda su severidad(6).

El uso de equipos de protección corporal (monos, batas, delantales, capuz, polainas, etc.) en la exposición a agentes biológicos en fluidos corporales junto con 
otros equipos de protección, parece reducir la tasa de contagios aunque los estudios son escasos i poco consistentes ${ }^{(7)}$.

Como protección del cuerpo, en el caso de riesgo bajo de exposición a agentes biológicos peligrosos se puede optar por bata entendida como EPI (con o sin polainas), mientras que, para exposiciones de alto riesgo, hay que emplear vestido/ mono integral preferentemente con capucha o capelina para proteger la cabeza y polainas hasta media pierna para cubrir los pies. En el caso de ropa de protección parcial, como batas, el trabajador debe llevar debajo la ropa de trabajo; mientras que en el caso de vestido/mono integral, debajo de éste debe llevar el mínimo de ropa imprescindible y preferentemente transpirable ${ }^{(3)}$. A la hora de elegir el tipo de EPI a utilizar para proteger el cuerpo, hay que tener en cuenta la magnitud del riesgo de exposición en función de la tarea a realizar, la dificultad para retirarse de forma segura el equipo (escoger el equipo que protege más adecuadamente según el nivel de riesgo y que es más fácil de retirar) y la comodidad/traspiración del equipo tal que proteja de forma adecuada con la mínima incomodidad.

Las batas consideradas EPI, deben ser bata larga cerrada atrás y con puños ajustables, con marcado CE categoría III. Las batas, al igual que las polainas, capuz, maguitos de antebrazos y los monos/trajes integrales de protección, deberán estar testadas según la norma UNE-EN 14605 y deberán cumplir con la clasificación adecuada al nivel de riesgo, en los ensayos de barrera contra la penetración de agentes biológicos según la norma UNE-EN 14126 (ISO 16604: resistencia a la penetración de patógenos transmisibles por sangre -bacteriófagos- bajo presión hidrostática, ISO 22610: resistencia a la penetración de agentes biológicos por contacto mecánico con sustancias que contienen líquidos contaminados, ISO/DIS 22611: resistencia a la penetración de aerosoles líquidos contaminados biológicamente, y ISO 22612: resistencia a la penetración de partículas sólidas contaminadas biológicamente)(3).

En situaciones de emergencia (brotes epidémicos) o de dificultades de mercado, en el que los fabricantes o suministradores puedan presentar problemas de suministro de estos equipos de protección individual, se pueden utilizar, de manera excepcional, batas certificadas únicamente como producto sanitario que hayan sido testadas por su resistencia a la penetración de fluidos, de acuerdo con las normas UNE-EN 13795 (nivel alto) y ASTM F1671, o bien la norma ANSI/AAMI PB70 (niveles 4 o 3). Es importante remarcar que las batas quirúrgicas de ropa no impermeable u otras batas que no cumplan estos requisitos puede que no confieran la protección mínima necesaria(3).

En muchas ocasiones, a pesar de que los equipos de protección corporal utilizados han superado las pruebas para ser certificados como EPIs, su diseño de patronaje (forma de cierre a nivel de cuello, tamaño de goma en los puños, longitud de las cintas de sujeción, existencia i tamaño de pestañas en las zonas de cierre,...) puede favorecer o dificultar una puesta o retirada segura del equipo o puede permitir una mejor o peor combinación con otros equipos (capuz, gafas de protección, mascarilla autofiltrante,...), pero no existen estudios comparativos en la eficacia de protección de los diferentes diseños. 
Hay que tener presente que para los monos integrales hay tallas normalizadas de acuerdo con las dimensiones del cuerpo humano pero no están directamente asociadas a las referencias de las casas comerciales ( $\mathrm{S}, \mathrm{M}, \mathrm{L}$, etc.). Esto implica que, para escoger la talla de los monos, se tendrán en cuenta las dimensiones corporales a las que se ajusta, que deben ser indicadas igualmente por el fabricante en la etiqueta de la pieza y del folleto informativo(3). Es necesario disponer de las tallas adecuadas a las características físicas de los trabajadores de cada centro de trabajo, en número suficiente para que no haya carencias de equipos de protección tanto para la asistencia de casos como para las sesiones de formación práctica para entrenar al trabajador en la colocación y la retirada correcta de los equipos. Cada centro de trabajo debe evaluar el stock mínimo de estos equipos para cubrir estas necesidades asistenciales y de formación, teniendo presente que los equipos destinados a formación pueden ser reciclados hasta que su grado de deterioro los haga inapropiados ${ }^{(3)}$.

Distintos organismos y centros asistenciales han elaborado metodologías para la puesta y retirada de equipos (CDC, Public Health England, entre otros), pero no se dispone de estudios comparativos concluyentes sobre errores o contaminaciones durante la retirada según las distintas metodologías. En éste sentido, sería interesante armonizar las distintas metodologías y realizar estudios comparativos de su eficacia.

Cuestiones de base organizacional como procedimientos breves, específicos sin ambigüedades y estables en el tiempo (sin cambios constantes), así como métodos de capacitación que tienen en cuenta las responsabilidades clínicas pueden influir en la eficacia de los EPIs y en la adherencia de uso por parte del trabajador sanitario $^{(8)}$.

Disponer de instalaciones o espacios adecuados para la puesta y retirada de EPIs (dimensiones adecuadas, instalaciones específicas de aislamiento de pacientes, ventilación adecuada, antesalas, duchas, instalaciones para lavarse las manos, instalaciones de descontaminación de superficie,...) y tallas o diseños adecuados a las diferentes necesidades según el nivel de riesgo puede influir en su eficacia(8).

Las experiencias vividas, como la evidencia cercana de un contagio, o la presión de grupo para el cumplimiento de protocolos pueden aumentar la adherencia a cumplir los procedimientos de aislamiento para el control de la infección. Mientras que una actitud complaciente del grupo hacia el cumplimiento de protocolos para el control de la infección en el lugar de trabajo, la percepción de que el uso del EPI por el trabajador sanitario puede ser estigmatizante para los pacientes y la incomodidad al usar el EPI, se han identificado como barreras para conseguir buenos niveles de adherencia al uso del EP|(8,9).

Los diseños de los EPIs, cuando es necesaria la combinación de varios de ellos, pueden condicionar la eficacia de los mismos, en tanto en cuanto su integración puede facilitar o dificultar la retirada conjunta de los mismos; a la vez que, ciertas combinaciones pueden potenciar la eficacia de unos sobre otros. Por ejemplo, el uso de una pantalla facial "protege" a un EPI respiratorio de salpicaduras con fluidos respiratorios, hecho que, facilita una retirada más segura de la mascarilla. 
Las publicaciones sobre metodología de formación a los trabajadores sanitarios para su correcta capacitación, aunque tienen baja consistencia metodológica, evidencian mayoritariamente que la adherencia al uso de EPIs de protección corporal es baja (alrededor del 77\% de las exposiciones), y que parece mejorar cuando se realizan cursos formativos (tanto de entrenamiento activo -realizando personalmente puesta i retirada de equipos- como pasivo -visualizando videos o representaciones de puesta i retirada-) respecto a la ausencia de entrenamiento al respecto(10).

Respecto a la retirada segura de equipos de protección, el 15,4\% de los encuestados se autoevaluó como que realiza retiradas seguras, el $63 \%$ con algún riesgo y el $22 \%$ con riesgo, y no se detectaron diferencias significativas entre los que habían recibido entrenamiento y los que no lo recibieron ${ }^{(10)}$.

No existen evidencias suficientes respecto a si metodologías de capacitación tradicionales son mejores o no respecto a metodologías con entrenamiento activo, apoyo con folletos informativos o simulaciones por computación pero un estudio controlado antes/después encontró que los participantes que recibieron educación de seguridad basada en entrevistas grupales motivacionales obtuvieron puntuaciones más altas en una lista de verificación que mide el uso de EPI (DM 2,95; IC del 95\%: 1,93 a 3,97) que los trabajadores de control que recibieron sesiones educativas tradicionales ${ }^{(11)}$.

Existe evidencia de muy baja calidad de que las intervenciones conductuales, es decir, la educación y la capacitación, puedan tener o no algún efecto sobre la frecuencia o uso correcto del equipo de protección respiratoria en los trabajadores, pero algunos estudios que comparan sesiones de formación basadas en entrevistas grupales motivacionales ven diferencias significativas en el comportamiento post-motivacional respecto a formaciones tradicionales ${ }^{(11)}$. Podría decirse que el cumplimiento correcto en el uso de EPI además de necesitar una capacitación teórico-práctica requiere también una acción motivacional que genere un cambio de actitud frente al uso del EPI.

En la actualidad no hay estudios suficientes para poder determinar, con un alto grado de evidencia científica, qué tipo de EPI protege mejor, cuál es la mejor manera de ponérselos y quitárselos ni cómo capacitar a los trabajadores sanitarios en el uso adecuado del equipamiento, requiriéndose una normalización de las combinaciones de equipos, más estudios comparativos sobre la eficacia de las distintas variantes metodológicas para poner y retirarse equipos y estudios comparativos sobre metodologías regladas de capacitación de los trabajadores sanitarios. 


\section{Bibliografía}

1. Verbeek JH, Rajamaki B, ljaz S, Sauni R, Toomey E, Blackwood B et al. Personal protective equipment for preventing highly infectious diseases due to exposure to contaminated body fluids in healthcare staff. Cochrane Database Syst Rev. 2020;4(4):CD011621. Update in: Cochrane Database Syst Rev. 2020;5:CD011621.

2. Burch J, Bunt C (on behalf of Cochrane Clinical Answers Editors). Which type of personal protective equipment (PPE), and which interventions to increase PPE use by healthcare workers, help reduce the spread of highly infectious diseases? Cochrane Clinical Answers. 2020. [Citado: 14 Jul, 2020.] Disponible en: https://doi. org//10.1002/cca.3056

3. Burch J, Hammerschmidt J (on behalf of Cochrane Clinical Answers Editors). What are the organizational, environmental, and individual barriers and facilitators affecting healthcare workers' adherence to infection prevention and control (IPC) guidelines for respiratory infectious diseases? Cochrane Clinical Answers. 2020. [Citado: 14 Jul, 2020.] Disponible en: https://doi.org/10.1002/cca.3067

4. Luong Thanh BY, Laopaiboon M, Koh D, Sakunkoo P, Moe H. Behavioural interventions to promote workers' use of respiratory protective equipment. Cochrane Database Syst Rev. 2016;12(12):CD010157.

5. Instituto Nacional de Seguridad e Higiene en el Trabajo. Guía Técnica para la utilización por los trabajadores de Equipos de Protección Individual. 2a edición, 2012. [Citado: 14 Jul, 2020.] Disponible en: https://www.insst.es/ documents/94886/203536/Gu\%C3\%ADa+t\%C3\%A9cnica+para+la+evaluaci\%C3\%B3n+y+prevenci\%C3\%B3n+de+los+riesgos+para+la+utilizaci\%C3\%B$3 n+$ por+los+trabajadores+en+el+trabajo+de+equipos+de+protecci\%C3\%B3n+individual/c4878c11-26a0-4108-80fd-3ecbef0aee38

6. Centro Nacional de Medios de Protección. Montes Mayorga M, coordinador. Equipos de protección individual (EPI). Aspectos generales sobre su comercialización, selección y utilización. INSHT. [Citado: 14 Jul, 2020.] Disponible en: https:// www.insst.es/documents/94886/203536/Gu\%C3\%ADa+INSST-EPI+Comercializaci\%C3\%B3n\%2C+selecci\%C3\%B3n+y+utilizaci\%C3\%B3n/28278487-9e2e-4b05 -9e8f-32684578f09e

7. Portillo García-Pintos J. Selección y uso de los Equipos de Protección Individual. Ficha de Divulgación Normativa. INSHT, 2010. [Citado: 14 Jul, 2020.] Disponible en: https://www.insst.es/documents/94886/371286/FDN-07+Selecci\%C3\%B3n+y+uso+de+los+Equipos+de+Protecci\%C3\%B3n+Individual+-+A\%C3\%B1o+2010+\%28vigente\%29/5ffdc9c1-1e20-4f60-b745-2590f96c4146

8. Portillo García-Pintos J. Comercialización de los Equipos de Protección Individual. Ficha de Divulgación Normativa. INSHT. [Citado: 14 Jul, 2020.] Disponible en: $\quad$ https://www.insst.es/documents/94886/371286/FDN-04+Comercializaci\%C3\%B3n+de+los+Equipos+de+Protecci\%C3\%B3n+individual+-+A\%C3\%B1o+1996+\%28vigente\%29/43ff215f-dc16-439e-9a4e-4527cb63c861 
9. Generalitat de Catalunya. de Montserrat i Nonó J, Juanola Pagès E, Inglés Torruella J, coordinadores. Guia d'actuació per a la prevenció de riscos laborals davant d'agents biològics perillosos altament transmissibles en centres sanitaris. 2017. [Citado: 14 Jul, 2020.] Disponible en: https://treball.gencat.cat/web/.content/09_-_seguretat_i_salut_laboral/documents/04_-_riscos_i_condicions_de_treball/riscos_laborals/Risc_biologic/GUIA-PRL-AGENTS-BIOLOGICS.pdf

10. Hersi M, Stevens A, Quach P, Hamel C, Thavorn K, Garritty C et al. Effectiveness of Personal Protective Equipment for Healthcare Workers Caring for Patients with Filovirus Disease: A Rapid Review. PLoS One. 2015;10(10):e0140290.

11. European Commission. Guide to application of the PPE Directive 89/686/EEC. 2015. [Citado: 14 Jul, 2020.] Disponible en: https://www.insst. es/documents/94886/203536/Guidelines+on+the+application+of+council+directive+89-686-EEC+of+21+December+1989+on+the+approximation+of+the+laws+of+the+member+states+relating+to+personal+protective+equipment/47b06ebf-c283-40ab-8f4d-aacffe2a9 